\title{
Patients' Educational Program Could Improve Azathioprine Adherence in Crohn's Disease Maintenance Therapy
}

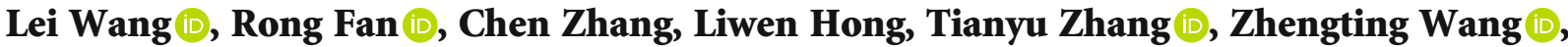 \\ and Jie Zhong
}

Department of Gastroenterology, Ruijin Hospital, Shanghai Jiaotong University School of Medicine, Shanghai, China

Correspondence should be addressed to Zhengting Wang; zhengtingwang@shsmu.edu.cn and Jie Zhong; jimmyzj64@hotmail.com

Received 23 November 2019; Accepted 4 April 2020; Published 20 April 2020

Academic Editor: Walter Fries

Copyright $(2020$ Lei Wang et al. This is an open access article distributed under the Creative Commons Attribution License, which permits unrestricted use, distribution, and reproduction in any medium, provided the original work is properly cited.

\begin{abstract}
Aim. To determine the risk factors of nonadherence to azathioprine (AZA) maintenance therapy for Crohn's disease (CD) and to evaluate the influence of patients' educational program on adherence to AZA maintenance therapy. Methods. Patients receiving AZA as maintenance therapy for CD were enrolled. Demographic data, clinical data, and usage information were collected. Univariate and multivariate analyses were performed to identify the risk factors of nonadherence. Then, patients' educational program was conducted. One year after the program, the improvements in adherence and relapse rates were compared between educational and noneducational groups. Results. A total of $378 \mathrm{CD}$ patients receiving AZA as maintenance therapy were enrolled from September 2008 to September 2018. Nonadherence occurred in 43.9\% (166/378) of patients. Univariate analysis revealed that young age, education, alcoholism, anxiety, depression, concern belief, and lack of necessity belief and AZA knowledge were risk factors of nonadherence $(P<0.05)$. Multivariate logistic regression showed that anxiety (OR 6.244, 95\% CI 2.563-15.213), depression (OR 3.801, 95\% CI 1.281-11.278), and concern belief (OR 19.531, 95\% CI 3.393-120.732) were independent risk factors of nonadherence. Necessity belief (OR 0.961, 95\% CI 0.925-0.999) and AZA knowledge (OR 0.823, 95\% CI 0.758-0.903) were protective factors of adherence. One year after the AZA educational program, the necessity belief, AZA knowledge, and adherence of the educational group significantly improved $(P<0.05)$. Concern belief was significantly lower in the educational group than that in the noneducational group $(P<0.05)$. Moreover, the noneducational group suffered significantly higher endoscopic relapse rates than that the educational group $(15.9 \%$ vs. $30.1 \%, P=0.035)$. Conclusions. Nonadherence occurred frequently in CD patients receiving AZA maintenance therapy. Educational programs could improve patients' adherence mainly by promoting their beliefs and knowledge of AZA and could reduce relapse rates during treatment.
\end{abstract}

\section{Introduction}

Crohn's disease $(\mathrm{CD})$ is a chronic inflammatory disease that is characterized by periods of remission and relapse and has an unpredictable course [1]. CD treatment includes two different phases: induction and maintenance of remission [2]. For most CD patients, the duration of maintenance therapy far outweighs that of induction therapy. Unlike Western countries, immunosuppressants, such as azathioprine (AZA), remain a mainstay in long-term maintenance therapy in developing countries, including China, where biological agents are not covered by medical insurance in most areas [3-5].
Medication nonadherence occurs in $30 \%-45 \%$ of patients with inflammatory bowel disease (IBD) $[6,7]$ and is associated with disease exacerbation, poor clinical outcomes, and increased economic burden [8, 9]. Improving adherence during maintenance therapy is of great importance in long-term CD management [10]. However, nonadherence to AZA maintenance therapy for $\mathrm{CD}$ has not been fully explored, especially in China. Further identifying the risk factors of nonadherence and investigating potential ways to improve adherence may be considerably important in $\mathrm{CD}$ maintenance therapy. In this study, we analyzed the medication adherence of $\mathrm{CD}$ patients to AZA maintenance therapy and determined the influence of educational programs on adherence and disease outcomes. 


\section{Methods}

2.1. Patient Enrollment. The institutional ethics board approved our study. Informed consent was acquired from all patients. This was a single-center cross-sectional and observational study. CD patients receiving AZA maintenance therapy for at least 6 months from September 2008 to September 2018 were enrolled. The diagnosis of CD was based on morphological (radiological, endoscopic, or surgical findings) and pathological criteria suggesting focal, asymmetrical, transmural, or granulomatous features [11]. Each patient experienced remission with Crohn's Disease Activity Index $(\mathrm{CDAI})<150$ through induction therapy with either corticosteroids or biologic agents. AZA doses were adjusted in accordance with side effects and blood tests in a stepwise manner to reach the maximal tolerated dose $(1.0-1.5 \mathrm{mg} / \mathrm{kg} /$ day) [12]. Patients receiving other concomitant treatment drugs, such as methotrexate, 5aminosalicylic acid, and corticosteroids, were excluded.

2.2. Assessment of AZA Adherence. AZA adherence was evaluated by using the Medication Adherence Report Scale (MARS), a four-item version of the questionnaire used in IBD [13]. Each question has a five-point scale and produces a score between 4 and 20. Similar to other studies, this work defined adherence as MARS 17 to 20.

2.3. Assessment of AZA Medication Beliefs. Beliefs about AZA medication were evaluated by using the Beliefs about Medicines Questionnaire (BMQs) [14]. The BMQs included two parts with five-point Likert scales: the belief of medication necessity and concerns about potential adverse effects. Each part involved five-item questionnaires with scores ranging from 5 to 25. Higher scores indicated greater belief of medication necessity or concerns about potential adverse effects. Belief of necessity and concerns about potential adverse effects were separately calculated, with scores of 15 to 25 defined as high medication beliefs or concerns. Medication acceptance was defined as having high necessity and low concern scores, which may be correlated with improved medication adherence.

2.4. Assessment of AZA Knowledge. AZA knowledge was evaluated with a 10 -item questionnaire designed specifically for Chinese patients (see Supplement). The questionnaire provided 1 point for each question. Total scores ranged from 0 to 10 with higher scores indicating better AZA knowledge. This questionnaire included treatment indication, dose, cessation, side effects, surveillance, and pregnancy and was believed to reflect the understanding and knowledge of AZA.

2.5. Analysis of Nonadherence to AZA Therapy. Demographic data, including gender, age, marriage, offspring, education, family income, disease cost, smoking, and alcoholism, were collected and compared to determine the risk factors for nonadherence. Clinical characteristics included disease duration, age of onset, disease location, disease behavior, perianal disease, CD-related surgery, anxiety, and depression. AZA usage information, such as AZA dosage, duration, necessity beliefs, concern beliefs, AZA knowledge, and side effects, was also recorded. Univariate and multivariate analyses were performed to determine the risk factors of nonadherence.

2.6. AZA Education and Its Impact on CD Maintenance Therapy. During October 2018 to September 2019, we conducted an AZA educational program for patients. This program was carried out by physicians of our department and consisted of face-to-face classes, on-line classes, and WeChat push. The main contents of this program focused on medical treatment and surveillance of CD under AZA treatment. Nonadherent patients were advised to participate in this program. After 1 year, we compared the psychological status, dosage, medication beliefs, AZA knowledge, adherence, side effects, and relapse rates between educational and noneducational groups. Surgical relapse meant that patients underwent bowel surgery due to CD-related complications. Clinical relapse was defined as CDAI > 150, and endoscopic relapse was regarded as Simple Endoscopic Score for Crohn's Disease exceeding 2 compared with the baseline [15]. We thus determined the impact of AZA education on CD maintenance therapy.

2.7. Statistical Analysis. Statistical analysis was performed using SPSS 22.0 (SPSS, Inc., Chicago, IL). Continuous variables are displayed as means \pm standard deviations and compared using Student's $t$ test, Mann-Whitney test, or one-way analysis of variance. Categorical variables were compared using Fisher's exact test or Chi-square test. Multivariate analysis was performed with logistic regression to identify factors associated with nonadherence using covariates found to be significant by univariate analysis. Statistical significance was regarded as $P<0.05$.

\section{Results}

3.1. Univariate Analysis of the Risk Factors of Nonadherence to AZA Maintenance Therapy for CD. A total of $378 \mathrm{CD}$ patients who received AZA as maintenance therapy from September 2008 to September 2018 were enrolled in our study. Nonadherence occurred in $43.9 \%$ (166/378) of patients. Univariate analysis revealed that among demographic parameters, young age, high educational level, and alcoholism were risk factors of nonadherence $(P<0.05)$ (Table 1). Gender, marriage status, offspring, family income, disease costs, and smoking did not significantly differ between the adherence and nonadherence groups. Moreover, the nonadherence group had significantly higher levels of anxiety and depression than the adherence group $(P<0.05)$ (Table 1). Other clinical parameters, such as disease duration, age of onset, disease location, disease behavior, perianal disease, and CD-related surgery, did not significantly differ between the two groups. Furthermore, we found that patients' low necessity belief and AZA knowledge and high concern belief were risk factors of nonadherence to AZA usage $(P<0.05)$ (Table 1$)$. AZA duration and side effects were not associated with patients' adherence.

3.2. Multivariate Logistic Regression on the Risk Factors of Nonadherence to AZA Maintenance Therapy for CD. We 
TABLE 1: Univariate analysis of risk factors for nonadherence in AZA maintenance.

\begin{tabular}{|c|c|c|c|}
\hline Features & Adherence $(n=212)$ & Nonadherence $(n=166)$ & $P$ value \\
\hline Male sex & $130(61.3)$ & $89(53.6)$ & 0.132 \\
\hline Age & $33.6 \pm 9.1$ & $29.7 \pm 11.9$ & 0.034 \\
\hline Married & $108(50.9)$ & $71(42.8)$ & 0.114 \\
\hline Offspring & $92(43.4)$ & $72(43.4)$ & 0.996 \\
\hline Education & & & $<0.001$ \\
\hline Primary school & $10(4.7)$ & $5(3.0)$ & \\
\hline Secondary school & $31(14.6)$ & $10(6.0)$ & \\
\hline High school & $73(34.4)$ & $40(24.1)$ & \\
\hline College & $93(43.9)$ & $90(54.2)$ & \\
\hline Postgraduate & $5(2.4)$ & $21(12.7)$ & \\
\hline Family income per month & & & 0.614 \\
\hline$>10$ thousand USD & $20(9.4)$ & $17(10.2)$ & \\
\hline 5-10 thousand USD & $30(14.2)$ & $21(12.7)$ & \\
\hline 2-5 thousand USD & $58(27.4)$ & $49(29.5)$ & \\
\hline 1-2 thousand USD & $79(37.3)$ & $52(31.3)$ & \\
\hline$<1$ thousand USD & $25(11.8)$ & $27(16.3)$ & \\
\hline Cost of disease per year & & & 0.328 \\
\hline$>10$ thousand USD & $51(24.1)$ & $34(20.5)$ & \\
\hline 5-10 thousand USD & $81(38.2)$ & $76(45.8)$ & \\
\hline$<5$ thousand USD & $80(37.7)$ & $56(33.7)$ & \\
\hline Smoking & $8(3.8)$ & $13(7.8)$ & 0.087 \\
\hline Alcoholism & $4(1.9)$ & $11(6.6)$ & 0.019 \\
\hline Disease duration (yrs) & $4.5 \pm 4.0$ & $4.3 \pm 4.0$ & 0.749 \\
\hline Age of onset & & & 0.509 \\
\hline$<17$ years old $(\mathrm{A} 1)$ & $18(8.5)$ & $19(11.4)$ & \\
\hline $17-40$ years old (A2) & $177(83.5)$ & $131(78.9)$ & \\
\hline$>40$ years old $(\mathrm{A} 3)$ & $17(8.0)$ & $16(9.7)$ & \\
\hline Location of lesions & & & 0.201 \\
\hline Ileum (L1) & $89(41.9)$ & $85(51.2)$ & \\
\hline Colon (L2) & $30(14.2)$ & $19(11.4)$ & \\
\hline Ileocolon (L3) & $93(43.9)$ & $62(37.3)$ & \\
\hline Behavior & & & 0.422 \\
\hline Nonstricture nonpenetrating & $144(67.9)$ & $102(61.4)$ & \\
\hline Stricture & $44(20.8)$ & $41(24.7)$ & \\
\hline Penetrating & $24(11.3)$ & $23(13.9)$ & \\
\hline Perianal disease & $69(32.5)$ & $49(29.5)$ & 0.528 \\
\hline $\mathrm{CD}$-related surgery & $42(19.8)$ & $39(23.5)$ & 0.387 \\
\hline Anxiety & $4.2 \pm 3.0$ & $7.6 \pm 4.0$ & $<0.001$ \\
\hline Depression & $5.1 \pm 3.9$ & $7.5 \pm 4.0$ & $<0.001$ \\
\hline \multicolumn{4}{|l|}{ AZA usage } \\
\hline Dosage $(\mathrm{mg} / \mathrm{d})$ & $68.4 \pm 35.2$ & $62.3 \pm 32.9$ & 0.082 \\
\hline Duration (months) & $33.8 \pm 24.7$ & $36.1 \pm 26.9$ & 0.157 \\
\hline Necessity belief & $17.9 \pm 2.1$ & $15.4 \pm 3.5$ & $<0.001$ \\
\hline Concerns belief & $14.6 \pm 3.0$ & $17.1 \pm 2.1$ & $<0.001$ \\
\hline Knowledge & $6.3 \pm 3.1$ & $4.4 \pm 2.7$ & 0.028 \\
\hline Side effect & $28(13.2)$ & $19(11.4)$ & 0.606 \\
\hline
\end{tabular}


TABLE 2: Multivariate analysis of risk factors for nonadherence in AZA maintenance.

\begin{tabular}{lccc}
\hline Variables & Odds ratio & $95 \%$ CI & $P$ value \\
\hline Anxiety & 6.244 & $2.563-15.213$ & $<0.001$ \\
Depression & 3.801 & $1.281-11.278$ & 0.016 \\
Necessity belief & 0.961 & $0.925-0.999$ & 0.045 \\
Concerns belief & 19.531 & $3.393-120.732$ & 0.003 \\
Knowledge & 0.823 & $0.758-0.903$ & 0.038 \\
\hline
\end{tabular}

conducted multivariate logistic regression for the parameters that had univariate significance. We found that on the one hand, anxiety (OR 6.244, 95\% CI 2.563-15.213), depression (OR 3.801, 95\% CI 1.281-11.278), and concern belief (OR 19.531, 95\% CI 3.393-120.732) were independent risk factors of nonadherence. On the other hand, necessity belief (OR 0.961, 95\% CI 0.925-0.999) and AZA knowledge (OR $0.823,95 \%$ CI $0.758-0.903)$ were protective factors of adherence (Table 2).

\subsection{Effects of AZA Education on AZA Usage in Nonadherent} Patients. As shown above, AZA belief and knowledge had a great impact on nonadherence. Thus, we carried out an AZA educational program to determine if it would be beneficial to improve adherence among patients. This program enrolled $38.0 \%(63 / 166)$ of nonadherent patients. After 1 year of follow-up, the levels of necessity belief, knowledge, and adherence of the educational group were significantly higher than those of the noneducational group $(P<0.05)$. By contrast, the concern belief of the educational group was significantly lower than that of the noneducational group $(P<0.05)$ (Table 3). For different dimensions of adherence in MARS, we found that "altered dose," "stopped medication," and "intentional miss" improved under AZA education, whereas "forgot medication" was similar between the two groups (Figure 1). Other parameters, such as anxiety, depression, dosage, and side effect, did not display significant differences between the AZA educational and noneducational groups.

\subsection{Effects of AZA Education on Disease Relapse in} Nonadherent Patients. We evaluated the clinical and endoscopic activity of nonadherent patients 1 year after the educational program. During 1 year of follow-up, 1.6\% (1/63) patients in the educational group experienced surgical relapse compared with $1.9 \%(2 / 103)$ in the noneducational group $(P=0.678)$. The clinical relapse rate of the AZA educational group was $9.5 \%(6 / 63)$, which was lower than that of the noneducational group $(15.5 \%, 16 / 103)$ without significant difference $(P=0.268)$. Moreover, the noneducational group suffered significantly higher endoscopic relapse rates than that the educational group $(15.9 \%$ vs. $30.1 \%, P=0.035)$ (Table 4) (Figure 2).

\section{Discussion}

Patients with CD have a high proportion of nonadherence likely because they are young and have a long quiescent period. Nonadherence to medication in maintenance treatment might directly lead to later flare-ups [8]. Demographic and clinical characteristics have some correlations with medication adherence, but most of them could not be intervening targets. The patients' knowledge and beliefs of medication, which could be influenced by their education, have tight associations with adherence [16]. Moreover, the role of patients' educational programs toward improving adherence remains controversial and has never been studied in CD maintenance therapy with immunosuppressants [17-19]. This study identified the risk factors of medication nonadherence in CD maintenance therapy with AZA and further explored the influence of patients' educational programs on adherence among patients with CD.

The nonadherence rate for maintenance therapy with AZA in our study was $43.9 \%$ with a MARS mean value of approximately 16.5, a value that is slightly higher than that reported for patients in Western countries $[6,7,20]$. This result can be attributed to the mild-to-moderate disease courses experienced by patients with CD in China, whereas patients in Western countries are more likely to experience severe activity [21]. Moreover, the patients in our study underwent AZA monotherapy as maintenance therapy. Quiescent disease may lead to the high rate of nonadherence.

We found that nonadherence was associated with the following demographic parameters: young age, high educational level, and alcoholism. This association might be due to the high self-righteousness and low medication necessity belief of young patients and patients with high educational levels. Alcoholism indicates poor self-management ability, which might lead to nonadherence. Moreover, clinical characteristics such as disease duration, age of onset, disease location, disease behavior, and perianal lesions did not have a significant impact on adherence. These findings were similar to those of other research [22-24]. Furthermore, high anxiety and depression levels, low necessity belief and AZA knowledge, and high concern belief were risk factors of nonadherence. These five parameters were further proven to be significant through multivariate analysis. This finding consequently indicated that adherence has more social and psychological instincts than physical-related instincts.

Patients' educational programs might modify medication beliefs and knowledge $[25,26]$. However, whether they can improve adherence and disease outcome and their impact on AZA usage in $\mathrm{CD}$ maintenance remain to be seen. In our study, we proved that AZA educational programs could significantly improve patients' adherence in three out of four aspects. These improvements might be attributed to the effect of the program on medication beliefs and knowledge. Our results proved that enhancing the patients' belief and AZA knowledge could improve adherence to AZA maintenance therapy for CD.

The AZA educational program may have a positive effect on disease outcome in CD maintenance $[27,28]$. This study showed that after 1 year of follow-up, the endoscopic relapse rate of patients in the educational group was significantly lower than that in the noneducational group. Meanwhile, the clinical relapse rate in the educational group was also considerably lower than that in the noneducational group. 
TABLE 3: The effects of AZA education on anxiety, depression, and its usage in nonadherence patients.

\begin{tabular}{lccc}
\hline Features & AZA educational group $(n=63)$ & AZA non-educational group $(n=103)$ & $P$ value \\
\hline Anxiety & $7.1 \pm 3.2$ & $7.5 \pm 4.1$ & 0.723 \\
Depression & $7.2 \pm 3.1$ & $7.3 \pm 4.3$ & \\
AZA usage & & & \\
Dosage (mg/d) & $71.6 \pm 34.9$ & $70.2 \pm 35.8$ & 0.674 \\
Necessity belief & $17.3 \pm 2.7$ & $16.2 \pm 4.1$ & 0.741 \\
Concern belief & $16.3 \pm 3.2$ & $17.3 \pm 2.6$ & 0.012 \\
Knowledge & $7.0 \pm 2.9$ & $4.7 \pm 2.7$ & $<0.001$ \\
Adherence & $17.3 \pm 2.0$ & $15.8 \pm 3.0$ & $<0.001$ \\
Side effect & $8(12.7)$ & $15(14.6)$ & 0.736 \\
\hline
\end{tabular}

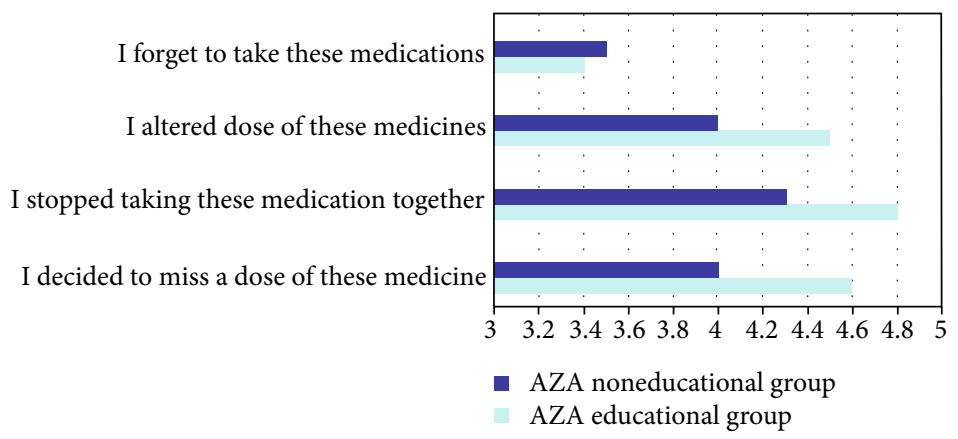

FIgURE 1: The impact of AZA educational program on different aspects of MARS.

TABLE 4: The effects of AZA education on disease relapse in nonadherence patients.

\begin{tabular}{lccc}
\hline $\begin{array}{l}\text { Efficacy } \\
\text { evaluation }\end{array}$ & $\begin{array}{c}\text { AZA } \\
\text { educational } \\
\text { group }(n=63)\end{array}$ & $\begin{array}{c}\text { AZA } \\
\text { noneducational } \\
\text { group }(n=103)\end{array}$ & $\begin{array}{c}P \\
\text { value }\end{array}$ \\
\hline $\begin{array}{l}\text { Surgery relapse } \\
\text { Clinical relapse }\end{array}$ & $1(1.6)$ & $2(1.9)$ & 0.678 \\
$\begin{array}{l}\text { Endoscopic } \\
\text { relapse }\end{array}$ & $10(15.5)$ & $16(15.5)$ & 0.268 \\
\hline
\end{tabular}

These findings further proved that AZA education is important in CD maintenance therapy.

Our study has several limitations. First, this is a singlecenter study and lacks long-term follow-up. Second, although all nonadherent patients were invited to attend our educational program, a few participated in the program. This behavior might prevent us from assessing this intervention comprehensively. Also, we should pay additional attention to the adherence of patients who did not participate in this program to alert possible disease flares. In the future, a multicenter research with larger patient number and longer follow-up should be encouraged to validate the effect of the program.

In conclusion, nonadherence occurred frequently in $\mathrm{CD}$ patients undergoing AZA maintenance therapy in China. Anxiety, depression, low necessity belief and knowledge, and high concern belief were independent risk factors of AZA nonadherence. The educational program conducted in this study could improve patients' adherence mainly by

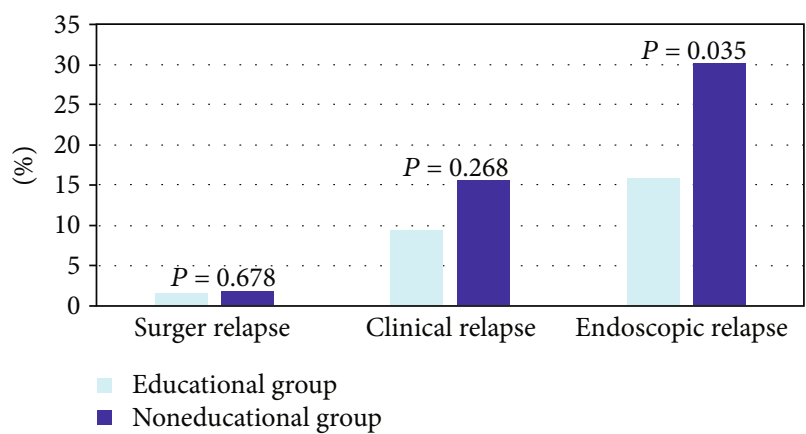

FIgURE 2: The effects of AZA educational program on disease relapse.

changing their beliefs and knowledge of AZA. Consequently, it could also reduce CD relapse rates during treatment.

\section{Data Availability}

All of the data in this manuscript are patients' clinical information, characteristics and several questionnaires that are collected by our researchers. These data are available by contacting the corresponding author through email once the article had been published.

\section{Ethical Approval}

The institutional ethics board of Ruijin Hospital approved our research. 


\section{Conflicts of Interest}

All the authors had no conflict of interest.

\section{Authors' Contributions}

All the authors approved this article for submission. Lei Wang and Rong Fan contributed equally to this work.

\section{Acknowledgments}

This study was supported by the National Natural Science Foundation of China (Nos. 81602558, 81670503, and 81970489), the Shanghai Committee of Science and Technology Foundation (No. 16411950402), and the Shanghai Health and Family Planning Committee (No. 201640112).

\section{Supplementary Materials}

It is the AZA knowledge questionnaire used to measure the level of patient's knowledge to AZA which has been described in the "Method" part of our manuscript. (Supplementary Materials)

\section{References}

[1] C. Mowat, A. Cole, A. Windsor et al., "Guidelines for the management of inflammatory bowel disease in adults," Gut, vol. 60, no. 5, pp. 571-607, 2011.

[2] A. Dignass, G. van Assche, J. O. Lindsay et al., "The second European evidence-based consensus on the diagnosis and management of Crohn's disease: current management," Journal of Crohn's \& Colitis, vol. 4, no. 1, pp. 28-62, 2010.

[3] J. P. Terdiman, C. B. Gruss, J. J. Heidelbaugh, S. Sultan, Y. T. Falck-Ytter, and AGA Institute Clinical Practice and Quality Management Committee, "American Gastroenterological Association Institute Guideline on the Use of Thiopurines, Methotrexate, and Anti-TNF- $\alpha$ Biologic Drugs for the Induction and Maintenance of Remission in Inflammatory Crohn's Disease," Gastroenterology, vol. 145, no. 6, pp. 1459-1463, 2013.

[4] R. Panaccione, A. H. Steinhart, B. Bressler et al., "Clinical practice guideline for management of luminal Crohn's disease: the Toronto consensus," Clinical Gastroenterology and Hepatology, vol. 7, 2019Epub ahead of print.

[5] T. Y. Zhang, Z. T. Wang, R. Fan et al., "The efficacy of infliximab monotherapy versus infliximab-azathioprine sequential treatment in Crohn's disease: experience from a tertiary medical center in China," BioMed Research International, vol. 2016, Article ID 8648307, 6 pages, 2016.

[6] C. A. Jackson, J. Clatworthy, A. Robinson, and R. Horne, "Factors associated with nonadherence to oral medication for inflammatory bowel disease: a systematic review," The American Journal of Gastroenterology, vol. 105, no. 3, pp. 525-539, 2010.

[7] M. J. Sewitch, M. Abrahamowicz, A. Barkun et al., "Patient nonadherence to medication in inflammatory bowel disease," The American Journal of Gastroenterology, vol. 98, no. 7, pp. 1535-1544, 2003.

[8] S. Kane, D. Huo, J. Aikens, and S. Hanauer, "Medication nonadherence and the outcomes of patients with quiescent ulcera- tive colitis," The American Journal of Medicine, vol. 114, no. 1, pp. 39-43, 2003.

[9] S. Kane and F. Shaya, "Medication non-adherence is associated with increased medical health care costs," Digestive Diseases and Sciences, vol. 53, no. 4, pp. 1020-1024, 2008.

[10] R. Horne, R. Parham, R. Driscoll, and A. Robinson, "Patients' attitudes to medicines and adherence to maintenance treatment in inflammatory bowel disease," Inflammatory Bowel Diseases, vol. 15, no. 6, pp. 837-844, 2009.

[11] F. Gomollón, A. Dignass, V. Annese et al., " 3 rd European evidence-based consensus on the diagnosis and management of Crohn's disease 2016: part 1: diagnosis and medical management," Journal of Crohn's \& Colitis, vol. 11, no. 1, pp. 325, 2017.

[12] L. F. Yu, J. Zhong, S. D. Cheng, Y. H. Tang, and F. Miao, "Lowdose azathioprine effectively improves mucosal healing in Chinese patients with small bowel Crohn's disease," Journal of Digestive Diseases, vol. 15, no. 4, pp. 180-187, 2014.

[13] R. Horne and J. Weinman, "Self-regulation and selfmanagement in asthma: exploring the role of illness perceptions and treatment beliefs in explaining non-adherence to preventer medication," Psychology \& Health, vol. 17, no. 1, pp. 17-32, 2002.

[14] R. Horne, J. Weinman, and M. Hankins, "The beliefs about medicines questionnaire: the development and evaluation of a new method for assessing the cognitive representation of medication," Psychology \& Health, vol. 14, no. 1, pp. 1-24, 1999.

[15] M. Daperno, G. D'Haens, G. van Assche et al., "Development and validation of a new, simplified endoscopic activity score for Crohn's disease: the SES-CD," Gastrointestinal Endoscopy, vol. 60, no. 4, pp. 505-512, 2004.

[16] C. P. Selinger, J. Eaden, D. B. Jones et al., "Modifiable factors associated with nonadherence to maintenance medication for inflammatory bowel disease," Inflammatory Bowel Diseases, vol. 19, no. 10, pp. 2199-2206, 2013.

[17] V. S. Conn and T. M. Ruppar, "Medication adherence outcomes of 771 intervention trials: systematic review and metaanalysis," Preventive Medicine, vol. 99, pp. 269-276, 2017.

[18] A. C. Moss, N. Chaudhary, M. Tukey et al., "Impact of a patient-support program on mesalamine adherence in patients with ulcerative colitis-a prospective study," Journal of Crohn's \& Colitis, vol. 4, no. 2, pp. 171-175, 2010.

[19] M. Elkjaer, M. Shuhaibar, J. Burisch et al., "E-health empowers patients with ulcerative colitis: a randomised controlled trial of the web-guided 'constant-care' approach," Gut, vol. 59, no. 12, pp. 1652-1661, 2010.

[20] A. B. Hawthorne, G. Rubin, and S. Ghosh, "Review article: medication nonadherence in ulcerative colitis-strategies to improve adherence with mesalazine and other maintenance therapies," Alimentary Pharmacology \& Therapeutics, vol. 27, no. 12, pp. 1157-1166, 2008.

[21] S. C. Ng, H. Y. Shi, N. Hamidi et al., "Worldwide incidence and prevalence of inflammatory bowel disease in the 21st century: a systematic review of population-based studies," Lancet, vol. 390, no. 10114, pp. 2769-2778, 2018.

[22] S. K. Tomar, S. Kedia, N. Singh et al., "Higher education, professional occupation, and upper socioeconomic status are associated with lower adherence to medications in patients with inflammatory bowel disease," JGH Open, vol. 3, no. 4, pp. 302-309, 2019. 
[23] I. Bruna-Barranco, A. Lué, C. J. Gargallo-Puyuelo et al., "Young age and tobacco use are predictors of lower medication adherence in inflammatory bowel disease," European Journal of Gastroenterology \& Hepatology, vol. 31, no. 8, pp. 948-953, 2019.

[24] R. D’Inca, P. Bertomoro, K. Mazzocco, M. G. Vettorato, R. Rumiati, and G. C. Sturniolo, "Risk factors for nonadherence to medication in inflammatory bowel disease patients," Alimentary Pharmacology \& Therapeutics, vol. 27, no. 2, pp. 166-172, 2008.

[25] M. V. Lenti and C. P. Selinger, "Medication non-adherence in adult patients affected by inflammatory bowel disease: a critical review and update of the determining factors, consequences and possible interventions," Expert Review of Gastroenterology \& Hepatology, vol. 11, no. 3, pp. 215-226, 2017.

[26] B. M. Waters, L. Jensen, and R. N. Fedorak, "Effects of formal education for patients with inflammatory bowel disease: a randomized controlled trial," Canadian Journal of Gastroenterology, vol. 19, no. 4, pp. 235-244, 2005.

[27] T. Moshkovska, M. A. Stone, R. M. Smith, J. Bankart, R. Baker, and J. F. Mayberry, "Impact of a tailored patient preference intervention in adherence to 5-aminosalicylic acid medication in ulcerative colitis: results from an exploratory randomized controlled trial," Inflammatory Bowel Diseases, vol. 17, no. 9, pp. 1874-1881, 2011.

[28] F. Depont, F. Berenbaum, J. Filippi et al., "Interventions to improve adherence in patients with immune-mediated inflammatory disorders: a systematic review," PLoS One, vol. 10, no. $12,2015$. 